\title{
REVIEW
}

\section{Axonal Outgrowth by Identified Neurons in the Spinal Cord of Zebrafish Embryos}

\author{
JOHN Y. KUWADA* $\uparrow$ AND ROBERT R. BERNHARDT* \\ ${ }^{*}$ Department of Biology and $\dagger$ Institute of Gerontology, University of Michigan, Ann Arbor, Michigan 48109
}

The spinal cord of early zebrafish embryos contains a small number of neurons per hemisegment. The earliest neurons are identifiable as individual neurons or small groups of homogeneous neurons and project growth cones that follow stereotyped, cell-specific pathways to their targets. These growth cones appear to bypass some axons but follow others during pathfinding, suggesting that they can distinguish among the different axons they normally encounter. Furthermore, identified growth cones exhibit cell-specific behaviors in apparent contact with the floor plate cells, which are found at the ventral midline of the early cord. These observations suggest the testable hypothesis that the floor plate may mediate multiple, cell-specific actions on identified growth cones in the zebrafish cord. One hypothesized action is inhibition of specific growth cones to prevent them from crossing the ventral midline. 1990 Academic Press, Inc.

\section{INTRODUCTION}

The behavior of individual growth cones during development has been delineated in a number of different nervous systems. These studies suggest that a number of different mechanisms guide growth cones including both attractive or positive $(1,3,5,8,12,17,19)$ and inhibitory $(6,7,14,15,18,20,21)$ interactions of growth cones with environmental cues. The most thorough investigations have utilized the relatively simple nervous system of the grasshopper embryo $(1,5)$. A detailed analysis of pathfinding by identified growth cones in these embryos allowed for the identification of cells and structures which could potentially influence them.

Detailed analyses of pathfinding are not limited to insect nervous systems, but can be accomplished in some vertebrate embryos. Recent investigations of pathfinding by the growth cones of identified motor neurons (4, 13) and identified spinal neurons $(2,8,10,11)$ demonstrated that the embryonic spinal cord of fish embryos is an attractive system for such detailed analyses. These studies have demonstrated the importance of cell-cell interactions for pathfinding by some neurons within the embryonic cord and identified a set of nonneuronal cells (floor plate cells) which potentially mediates both cellspecific attractive and inhibitory actions on identified spinal growth cones.

\section{ORGANIZATION OF THE SPINAL CORDS OF EARLY FISH EMBRYOS}

The spinal cords of fish embryos are simple enough to allow for a relatively complete and detailed characterization especially during the earliest periods of development. Since the early fish embryo is transparent and thin (approximately 50 to $100 \mu \mathrm{m}$ thick in the main trunk), the spinal neurons which are 5 to $15 \mu \mathrm{m}$ in diameter are easily visualized in the living embryo with a compound microscope outfitted with differential interference contrast optics. These neurons can be labeled retrogradely by application of horseradish peroxidase (HRP) or lipophilic dyes (diI and diO) into a spinal tract, labeled orthogradely with lipophilic dyes applied to their cell bodies, impaled under visual control with microelectrodes, and filled with HRP or Lucifer yellow (LY), and labeled with monoclonal antibodies which recognize embryonic neurons. These techniques allowed us to identify many of the neurons in the early cord and label embryonic neurons from the time they initially projected growth cones. Embryonic spinal neurons were identified on the basis of their unique locations, sizes, and morphologies.

The early (18-20 h) cord of zebrafish embryos consists of neuroepithelial cells, floor plate cells, and approximately 18 neurons per hemisegment (2). By this time 8 to 11 of these early neurons have projected growth cones and fall into six classes of identified neurons. These are 3 primary motor neurons (13), approximately 3 mechanosensory Rohon-Beard (RB) neurons, 1 primary commissural interneuron (CoPA), 0-2 secondary commissural interneurons (CoSA), 0-1 dorsal longitudinal interneuron (DoLA), and 1 ventral longitudinal interneuron (VeLD (2)) (Figs. 1 and 2). Additionally, there are approximately 20 floor plate cells, which make up the ventral floor of the cord, in each segment (10).

The RB neurons are the dorsal-most neurons in the embryonic cord and have a long ascending and descend- 


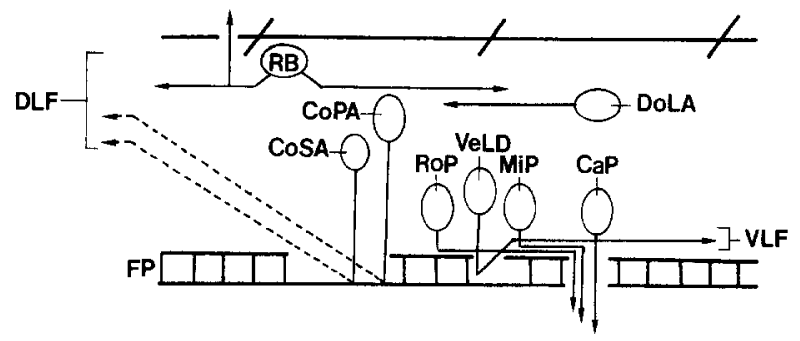

FIG. 1. A schematic summarizing the neuronal classes and major axonal tracts in the spinal cords of 18 - to 20 -h zebrafish embryos. The location along the dorsal/ventral axis and the relative size of cell bodies are as shown. See text for the distribution and soma position along the anterior/posterior axis and most of the abbreviations. In this and all other figures, unless otherwise noted, anterior is left, dorsal is up, the segment borders are indicated by diagonal lines, arrows at the ends of axons indicate that the axons extend further, and contralateral axons and somata are indicated by dashed lines. $\mathrm{RoP}, \mathrm{MiP}$, and $\mathrm{CaP}$ are the primary motor neurons and FP denotes the row of floor plate cells.

ing axon in a dorsal longitudinal fasciculus (DLF) as well as a peripheral axon which arises from the central axon to innervate the skin. The two classes of early commissural interneurons (CoPA and CoSA) are located in the dorsal cord and both have a single axon which courses ventrally and ascends in the contralateral DLF. CoPA and CoSA differ in a number of characteristics: there is usually a single CoPA and up to five CoSAs in each hemisegment in the larval cord; the CoPA soma is larger than the CoSA soma; CoPA neurons project growth cones several hours before CoSA neurons; CoPA neurons are tripolar and possess distinctive longitudinal dendrites; CoPA axons are long and terminate in the region of the diencephalon/mesencephalon border, while CoSA axons are considerably shorter; CoPA but not CoSA neurons are labeled by the anti-CON1 monoclonal antibody prior to $28 \mathrm{~h}(9)$. The DoLA neurons are relatively rare, located in the dorsal cord, and have an ipsilateral, ascending longitudinal axon in the DLF. The VeLD neurons are located in the ventral cord and have an ipsilateral, descending longitudinal axon in the ventral longitudinal fasciculus (VLF).

\section{DEVELOPMENT OF IDENTIFIED SPINAL NEURONS}

Each of the early spinal neurons projects the appropriate number of growth cones which extend along precise, cell-specific pathways to reach their termination sites in the CNS (11). These neurons do not project axons into every available pathway followed by retraction of the inappropriate axons. The specificity of early outgrowth is strikingly demonstrated by the growth cones of two of the early neurons that exhibit cell-specific behaviors in the vicinity of the ventral midline (10). The CoPA neuron projects a single growth cone from the ventral pole of the cell body at 16-17 $\mathrm{h}$ of development (Fig. 3A). After emerging from the soma, the growth cone extends ven- trally along the superficial surface of the cord between the endfeet of neuroepithelial cells and the lateral cel! bodies of neurons to reach the ventral midline by 18-19 h. After crossing the ventral midline the CoPA growth cone turns anterior and follows a diagonal pathway to the DLF. When the growth cone reaches the DLF it turns onto it and ascends. The VeLD neuron like the CoPA neuron projects a single growth cone from the ventral pole of its cell body (Fig. 3B). This growth cone extends toward the ventral midline but does not cross the midline; rather it turns posterior and descends in the VLF.

\section{IDENTIFIED GROWTH CONES BYPASS SOME AXONS AND APPEAR TO FOLLOW OTHERS}

Since the early cord of zebrafish embryos consists of a small number of cell types, the early spinal growth cones
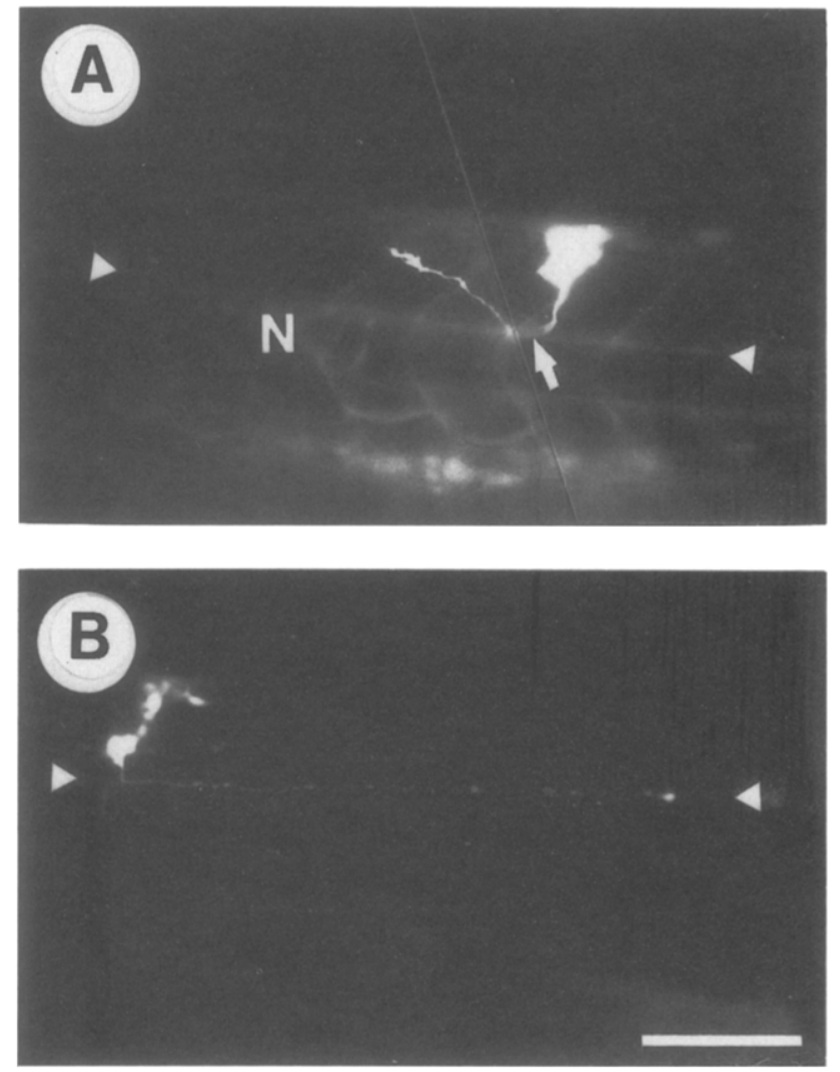

FIG. 2. Lateral view of identified spinal neurons in 20 -h embryos labeled by intracellular LY injections. In each case adjacent neuroepithelial cells were inadvertently labeled. We verified that the neuroepithelial cells were not actually part of the cell by backfilling neurons with diI and by applying MAbs which label embryonic neurons. (A) LY-filled CoPA neuron. This is a photomontage which displays the soma and the contralateral growth cone and axon in focus simultaneously. Arrow denotes the point where the axon crosses the ventral midline; triangles, ventral border of the cord; N, notochord. (B) LYlabeled VeLD neuron. Arrow denotes the VeLD soma which is ventral to the line of neuroepithelial cells the electrode passed through in order to impale the VeLD soma. Scale for A, $100 \mu \mathrm{m}$; for B, $200 \mu \mathrm{m}$. 
A
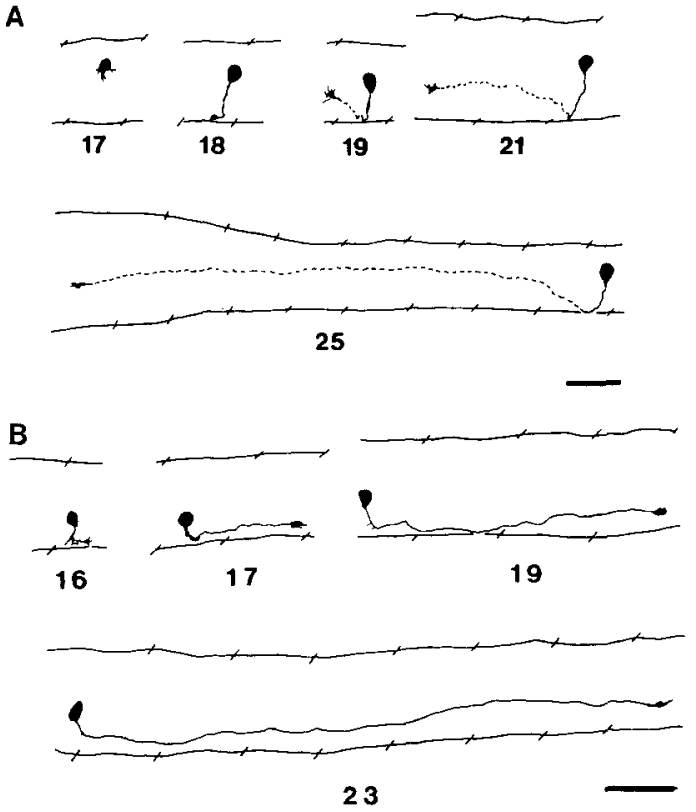

FIG. 3. Development of CoPA and VeLD neurons. Camera lucida drawings of LY-filled neurons at various stages in development seen in lateral views. The hour of development is indicated by numbers under each drawing. (A) CoPA neuron. In this and all subsequent figures the axon contralateral to the cell body is dashed. Contralateral growth cones are not dashed. Scale, $50 \mu \mathrm{m}$. (B) VeLD neurons. Note that the growth cone is initially projected from the ventral pole of the soma. Scale, $50 \mu \mathrm{m}$.

encounter a small set of identified neuronal somata and axons (10). For example, the DLF will run across the ventral portion of CoPA cell bodies in older embryos and, therefore, the CoPA growth cones could potentially extend longitudinally rather than ventrally. There are no barriers to longitudinal growth in this region at the time CoPA growth cones are initially projected since the growth cones of the other two classes of early dorsal neurons, the RB and DoLA neurons, freely extend longitudinally at this time (Fig. 4A). Likewise, in the ventral cord the CoPA growth cone bypasses the longitudinal axons of both ipsilateral (Fig. 4B) and contralateral VeLD neurons. On the other hand, identified growth cones appear to follow the longitudinal axons of homologous neurons. This can be seen from LY fills of neighboring CoPA neurons (Fig. 4C), neighboring VeLD neurons (Fig. 4D), and neighboring DoLA neurons. Labeling of CoPA axons and growth cones with the anti-CON1 antibody and VeLD axons and growth cones with discrete applications of diI into the VLF corroborated the LY injections.

These observations suggest that identified growth cones bypass some axons but follow others and, therefore, they can distinguish among the different axons they encounter. In this regard the behavior of commissural growth cones is especially intriguing. CoPA growth cones do not extend longitudinally in the ipsilateral
DLF, although other growth cones do (see above), but do ascend in the contralateral DLF. Similarly some CoSA growth cones bypass the CoPA longitudinal axons on the ipsilateral side, but turn onto the DLF containing CoPA axons on the contralateral side. These commissural growth cones, therefore, respond differently to longitudinal pathways depending on whether they have crossed the ventral midline or not. Finally, analysis of the local environment of identified growth cones rules out that nonspecific constraints such as mechanical barriers are responsible for directionality of axon outgrowth.

\section{THE FLOOR PLATE CELLS ARE THE SITE OF CELL-SPECIFIC BEHAVIORS BY IDENTIFIED GROWTH CONES}

As described above, the region of the ventral midline is particularly interesting since it is the site of cell-specific behaviors by the CoPA and VeLD growth cones. This region consists of a row of floor plate cells approximately three cells wide (10) (Fig. 5). These cells are presumably nonneuronal, extend no processes, and differ from the other neuroepithelial cells in shape. Additionally, the middle row of floor plate cells is antigenically different

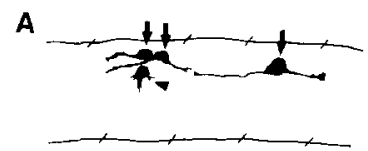

B
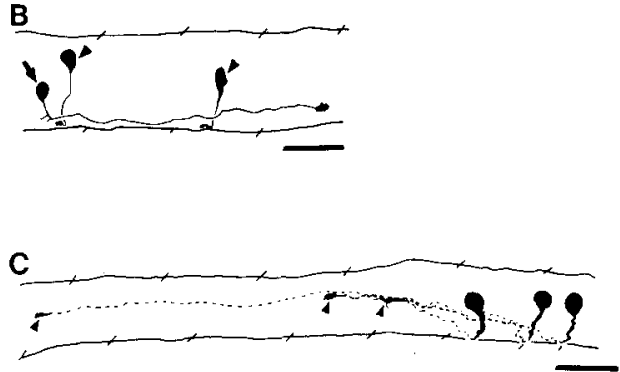

D

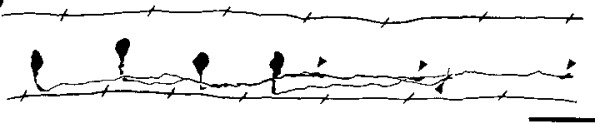

FIG. 4. Identified growth cones bypass the pathways of other identified growth cones but extend in apparent contact with the axons of the same neuronal class. Camera lucida drawings of LY-filled neurons. (A) RB (arrows) axons and growth cones are nearby a CoPA neuron (arrowhead) just projecting a growth cone at $17 \mathrm{~h}$. (B) Two CoPA growth cones have extended by the longitudinal axon of a nearby VeLD neuron (arrow) in a 19-h embryo. Scale for A and B, 50 $\mu \mathrm{m}$. (C) Three CoPA neurons from a 23-h embryo. Arrowhead, growth cone; scale, $50 \mu \mathrm{m}$. (D) Four VeLD neurons from a 24-h embryo; scale, $50 \mu \mathrm{m}$. 

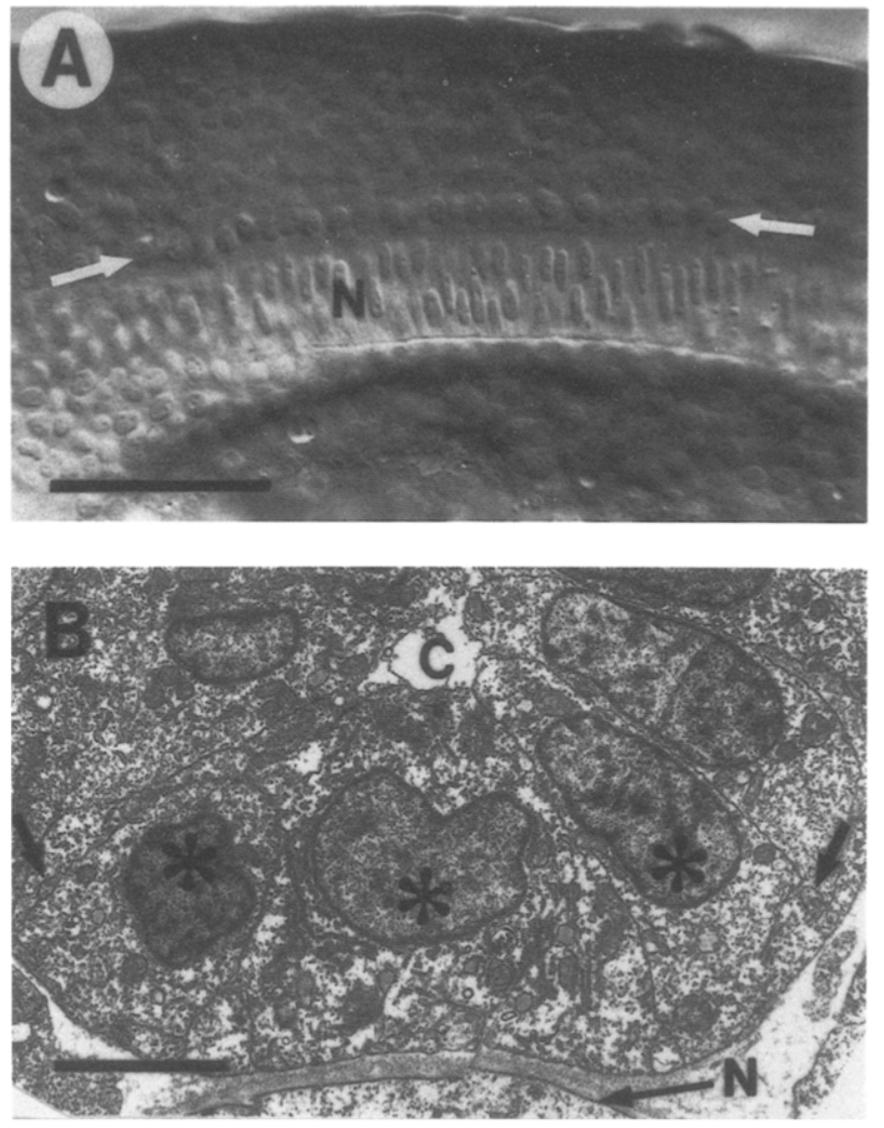

FIG. 5. Floor plate cells are identified cells which make up the ventral floor of the early cord. (A) DIC micrograph of the middle row of floor plate cells (arrows) seen in a sideview from a live 17-h embryo. Anterior is to the left and dorsal is up; $\mathrm{N}$, notochord; scale, $50 \mu \mathrm{m}$. (B) EM micrograph of the floor plate cells (asterisks) in cross section from a 20 -h embryo. Arrows point to two axonal profiles, which may represent VeLD axons, in contact with the lateral floor plate cells. C, central canal; $\mathrm{N}$, notochord; scale, $5 \mu \mathrm{m}$.

from other neuroepithelial cells (Charles Kimmel, personal communication). The floor plate cells are among the earliest cells to become distinguishable in the cord and are evident several hours prior to the first spinal growth cones.

Identified growth cones exhibit cell-specific behaviors in the immediate vicinity of the floor plate cells. CoPA growth cones extend between the floor plate cells and the basal lamina to cross the ventral midline. While in apparent contact with the floor plate cells CoPA growth cones turn anterior (Figs. 6A and 6B). In contrast, VeLD growth cones extend to the floor plate cells but do not cross the midline. Instead they turn posterior and extend caudally along the floor plate cells in the ipsilateral, ventral cord (Figs. 6C and 6D). In addition to the CoPA and VeLD growth cones, the growth cones of CoSA neurons and $\mathrm{CoB}$ neurons (commissural neurons with axons which bifurcate and extend both rostrally and caudally on the contralateral side) also exhibit cell-specific be- haviors at the floor plate. CoSA growth cones behave similarly to $\mathrm{CoPA}$ growth cones and $\mathrm{CoB}$ growth cones bifurcate soon after crossing the ventral midline.

These observations suggest that the floor plate may have multiple, cell-specific effects on identified spinal growth cones in the zebrafish embryo. More specifically our work has generated several interesting, testable hypotheses. (i) Floor plate cells attract some growth cones (CoPA, CoSA, CoB, and VeLD) to extend toward the ventral midline but not others (RB and DoLA). (ii) Floor plate cells induce the CoPA, CoSA, and $\mathrm{CoB}$ growth cones to cross and make cell-specific turns. (iii) Floor plate cells inhibit the VeLD growth cone from crossing the midline and instead induce it to turn posterior. (iv) Interactions with the floor plate cells may be necessary for commissural growth cones to turn onto the appropriate longitudinal pathway. These hypotheses can be tested by (i) laser ablation of floor plate cells, (ii) transplantation of floor plate cells to ectopic sites, and (iii) examination of Cyclops mutants in which the floor plate fails to develop (Charles Kimmel, personal communication).

\section{THE ROLE OF FLOOR PLATE CELLS FOR GUIDANCE OF SPINAL GROWTH CONES}

The floor plate plays a critical role in axonogenesis in the rat cord by attracting the growth cones of commissural neurons $(3,17)$. In the zebrafish embryo the floor plate cells are among the earliest cells that can be identified in the spinal cord and may have multiple, cell-specific actions on identified growth cones. Among the possible actions of the floor plate is inhibition of the ventrally directed VeLD growth cone to prevent it from crossing the ventral midline. The floor plate may also potentially keep other growth cones from crossing the midline. For example, in the second day of embryogenesis the circumferential classes of spinal neurons develop. Circumferential neurons have axons that initially run ventrally toward the floor plate but turn anterior or posterior in the ipsilateral ventral cord and never cross the ventral midline (11). Although at present we know little about the relationship between the floor plate and circumferential growth cones, it is possible that circumferential growth cones may also be inhibited from crossing the midline by the floor plate cells. These observations coupled with the fact that commissural growth cones readily cross the midline suggest that the hypothesized inhibitory actions of the floor plate are specific in zebrafish embryos. Like attractive or positive interactions between growth cones and environmental cues ( 5 , $8,12,19)$ and inhibitory interactions between growth cones and axons in vitro $(6,7,21)$, inhibitory actions on growth cones may also be specific in vivo. 

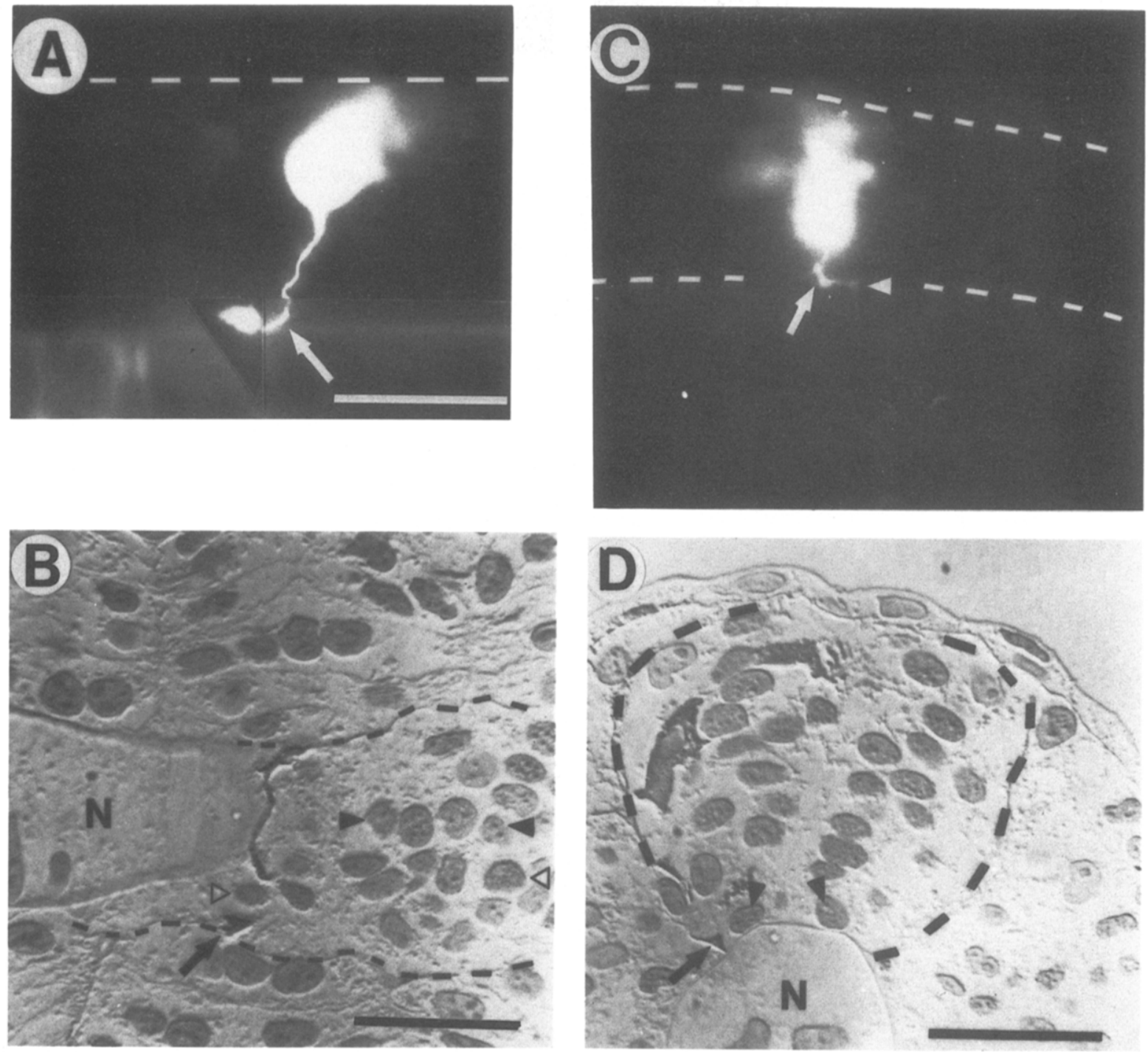

FIG. 6. The CoPA and VeLD growth cones both are initially ventrally directed and exhibit cell-specific behaviors in apparent contact with the floor plate. (A) Fluorescence micrograph of a lateral view of a LY-filled CoPA neuron with a growth cone just past the ventral midline from a 19-h embryo. This is a photomontage which displays the soma and the contralateral growth cone, which are at different focal planes, simultaneously. Arrow, point where the axon crosses the ventral midline; dashed line, dorsal border of the cord; ventral border of the cord is the faint line just ventral to the growth cone. (B) CoPA growth cones extend under the floor plate cells and turns anterior. Horizontal section showing that a LY/anti-LY-labeled CoPA growth cone (arrow) has turned anterior just past the lateral row of floor plate nuclei (open triangles) in an 18-h embryo. The CoPA axon can be seen crossing the floor of the cord in the middle of the micrograph. A gap between the axon and the growth cone appears because the portion of the axon just proximal to the growth cone was located in an adjacent section. On the left this section passed through the underlying notochord $(\mathrm{N})$. Axial muscles are on either side of the cord. Arrowheads, middle floor plate cells and ventral midline; dashed lines, lateral borders of the ventral cord; scale for A, $50 \mu \mathrm{m}$; scale for $\mathrm{B}, 25 \mu \mathrm{m}$. (C) Fluorescence photograph of a lateral view of a LY-filled VeLD growth cone which has just turned posterior at the ventral floor of the cord in a 16-h embryo. Arrowhead, leading edge of the growth cone; arrow, proximal part of the growth cone; dashed lines, dorsal and ventral outline of the cord. (D) VeLD growth cone extends to the floor plate cells and turns and extends posteriorly along the floor plate cells. Cross section showing the LY/anti-LY-labeled VeLD growth cone (arrow) extending posteriorly along the lateral floor plate cells (arrowheads denote their nuclei) in a 17-h embryo. The nucleus of a middle floor plate cell was not in this section. There is a lightly labeled unidentified neuron dorsal to the VeLD growth cone. Dashed lines, Iateral borders of the cord; $\mathrm{N}$, notochord; scale for $\mathrm{C}, 50 \mu \mathrm{m}$; scale for $\mathrm{D}, 25 \mu \mathrm{m}$.

\section{ACKNOWLEDGMENTS}

We thank A. Chitnis, N. Nguyen, L. Lindamer, and P. Knafl for contributing to the studies presented in this paper and for technical assistance. This work was supported by grants from NIH (NS24848), Office of the Vice-President for Research at the University of Michigan, and a Basil O'Connor Scholar Award from the March of Dimes Birth Defects Foundation to J.Y.K.

\section{REFERENCES}

1. Bentley, D., AND M. Caudy. 1983. Pioneer axons lose directed growth after selective killing of guidepost cells. Nature (London) 304: 65.

2. BernhardT, R. R., A. B. Chitnis, L. A. Lindamer, AND J. Y. KUWADA. 1990. Identification of spinal neurons in the embryonic and larval zebrafish. J. Comp. Neurol. Submitted for publication. 
3. DoDD, J., AND T. JESSELL. 1988. Axon guidance and the patterning of neuronal projections in vertebrates. Science 242: 692-699.

4. Ersen, J. S., S. H. PIKE, AND DEBU, B. 1989. The growth cones of identified motoneurons in embryonic zebrafish select appropriate pathways in the absence of specific cellular interactions. Neuron 2: 1097-1104.

5. Goodman, C. S., M. J. Bastiani, C. Q. Doe, S. DU LaC, S. L. HELFAND, J. Y. KuWADA, AND J. B. ThOMAS. 1984. Cell recognition during neuronal development. Science 225: 1271-1279.

6. KaPfHAMmer, J. P., B. E. GRUNEWALD, AND J. A. RAPER. 1986. The selective inhibition of growth cone extension by specific neurites in culture. J. Neurosci. 6: 2527-2534.

7. KAPFHAMMER, J. P., AND J. A. RAPER. 1987. Collapse of growth cone structure on contact with specific neurites in culture. $J$. Neurosci. 7: 201-212.

8. KUWADA, J. Y. 1986. Cell recognition by neuronal growth cones in a simple vertebrate embryo. Science 233: 740-746.

9. Kuwada, J. Y., A. B. Chitnis, and L. A. Lindamer. 1987. Stereotyped pathfinding by identified growth cones of the embryonic cord. Soc. Neurosci. Abstr. 13: 1220.

10. Kuwada, J. Y., R. R. Bernhardt, and A. B. Chitnis. Pathfinding by identified growth cones in the spinal cord of zebrafish embryos. $J$. Neurosci., in press.

11. KuwadA, J. Y., R. R. Bernhardt, and N. NGUYEN. 1990. Development of spinal neurons and tracts in the zebrafish embryo. $J$. Comp. Neurol. Submitted for publication.

12. LuMSDEN, A. G. S., AND A. M. DAviES. 1986. Chemotropic effect of specific target epithelium in the developing mammalian nervous system. Nature (London) 323: 538-539.
13. MYers, P. Z., J. EISEN, AND M. WESTERFIELd. 1986. Development and axonal outgrowth of identified motoneurons in the zebrafish. J. Neurosci. 6: 2278-2289.

14. SCHWAB, M. E., AND P. CARONI. 1988. Oligodendrocytes and CNS myelin are nonpermissive substrates for neurite growth and fibroblast spreading in vitro. J. Neurosci. 8: 2381-2393.

15. SILVER, J. 1984. Studies on the factors that govern directionality of axonal growth in the embryonic optic nerve and at the chiasm of mice. J. Comp. Neurol. 223: 238-251.

16. SNow, D. M., V. Lemmon, AND J. SiLver. 1989. Evidence for inhibition of axon outgrowth by keratan sulfate/chondroitin sulfate proteoglycan in vivo and in vitro. Soc. Neurosci. Abstr. 15: 332.

17. Tessier-Lavigne, M., M. Placzek, A. G. S. Lumsden, J. DodD, AND T. M. Jessell. 1988. Chemotropic guidance of developing axons in the mammalian central nervous system. Nature (London) 336: 775-778.

18. Tosney, K. W., AND L. T. LANDMEsSer. 1984. Pattern and specificity of axonal outgrowth following varying degrees of chick limb bud ablations. J. Neurosci. 4: 2518-2527.

19. TosnEy, K. W. 1987. Proximal tissues and patterned neurite outgrowth at the lumbosacral level of the chick embryo: Deletion of the dermamyotome. Dev. Biol. 122: 540-588.

20. Tosney, K. W. 1988. Proximal tissues and patterned neurite outgrowth at the lumbosacral level of the chick embryo: Partial and complete deletions of the somite. Dev. Biol. 127: 266-286.

21. Walter, J., S. Henke-Fahle, AND F. BonhoffFer. 1987. Avoidance of posterior tectal membranes by temporal retinal axons. Development 101: 909-913. 